\title{
A Detail-Preserving Scale-Driven Approach to Change Detection in Multitemporal SAR Images
}

\author{
Francesca Bovolo and Lorenzo Bruzzone, Senior Member, IEEE
}

\begin{abstract}
This paper presents a novel approach to change detection in multitemporal synthetic aperture radar (SAR) images. The proposed approach exploits a wavelet-based multiscale decomposition of the log-ratio image (obtained by a comparison of the original multitemporal data) aimed at achieving different scales (levels) of representation of the change signal. Each scale is characterized by a different tradeoff between speckle reduction and preservation of geometrical details. For each pixel, a subset of reliable scales is identified on the basis of a local statistic measure applied to scale-dependent log-ratio images. The final changedetection result is obtained according to an adaptive scale-driven fusion algorithm. Experimental results obtained on multitemporal SAR images acquired by the ERS-1 satellite confirm the effectiveness of the proposed approach.
\end{abstract}

Index Terms-Change detection, image analysis, multiscale image decomposition, remote sensing, synthetic aperture radar (SAR).

\section{INTRODUCTION}

C HANGE detection is a process that analyzes a pair of remote sensing images acquired on the same geographical area at different times in order to identify changes that may have occurred between the considered acquisition dates. Change-detection techniques have been used successfully in many applications, such as environmental monitoring [1], study on land-use/land-cover dynamics [2], analysis of forest or vegetation changes [3], [4], damage assessment [5], agricultural surveys [6], and analysis of urban changes [7], [8].

In recent years, several change-detection techniques have been proposed in the remote sensing literature for the analysis of images acquired by passive sensors. Less attention has been devoted to change detection in synthetic aperture radar (SAR) images. This is mainly due to the intrinsic complexity of SAR data, which require both an intensive preprocessing phase and the development of effective data analysis techniques capable of dealing with multiplicative speckle noise. Because of these two issues, end-users are less interested in SAR data for operational change-detection applications. However, despite the complexity of data processing, SAR sensors have important properties at the operational level, since they are capable of acquiring data in all weather conditions and are not affected by cloud cover or different sunlight conditions (see [9] for greater details on the importance and the properties of SAR data in change detection).

Manuscript received January 4, 2005; revised August 4, 2005. This work was supported by the Italian Ministry of Education, University, and Research.

The authors are with the Department of Information and Communication Technologies, University of Trento, I-38050 Trento, Italy (e-mail: lorenzo. bruzzone@ing.unitn.it).

Digital Object Identifier 10.1109/TGRS.2005.857987
In the literature, usually unsupervised change detection in SAR images is based on a three-step procedure [9]: 1) preprocessing; 2) pixel-by-pixel comparison of two images; and 3 ) image thresholding. The aim of preprocessing is to increase the SNR of the considered images (by reducing noisy speckle components), while preserving sufficient spatial details. Many adaptive filters for speckle reduction have been proposed, e.g., the Frost [10], Lee [11], Kuan [12], Gamma Map [13], [14], and Gamma WMAP [15] (i.e., the Gamma MAP filter applied in the wavelet domain) filters. Despite their spatial adaptive characteristic, which tends to preserve the signal's high-frequency information, filter applications often give the desired speckle reduction but also an undesired degradation of the geometrical details of the investigated scene. Pixel-by-pixel comparison is carried out according to a ratio (or a log-ratio) operator in such a way as to take into account the multiplicative model of the speckle. The decision threshold can be selected either with a manual trial-and-error procedure (according to the desired tradeoff between false and missed alarms) or with automatic techniques (e.g., by analyzing the statistical distribution of the ratio image, by fixing the desired false alarm probability [4], [16], or following a Bayesian minimum error decision rule [9]).

Depending on the kind of preprocessing applied to the multitemporal images, these techniques can achieve different tradeoffs between detail preservation and accuracy in the representation of homogeneous areas in change-detection maps. But these are contrasting properties, in other words high accuracy in homogeneous areas usually requires an intensive despeckling phase, which in turn degrades the geometrical details in the SAR images. This is due both to the filter's smoothing effect and to the removal of the speckle's informative components (which is related to the coherence properties of the SAR signal).

In order to address the above limitations of standard methods, in this paper we propose a scale-driven, adaptive approach to change detection in multitemporal SAR images. This is based: 1) on a multiscale decomposition of the log-ratio image; 2 ) on a selection of the reliable scales for each pixel (i.e., the scales at which the considered pixel can be represented without border problems) according to an adaptive analysis of its local statistics; and 3) on a scale-driven combination of the selected scales. In greater detail, we propose to perform the scale-driven combination by investigating three different strategies: a) fusion at the decision level by an "optimal" scale selection; b) fusion at the decision level of all reliable scales; and c) fusion at the feature level of all reliable scales. The rationale of the proposed method is to exploit only high-resolution levels in the analysis of the expected edge (or detail) pixels and to use also low-resolution levels in the processing of pixels in homogeneous areas. The proposed method thus exhibits both a high sensitivity to 


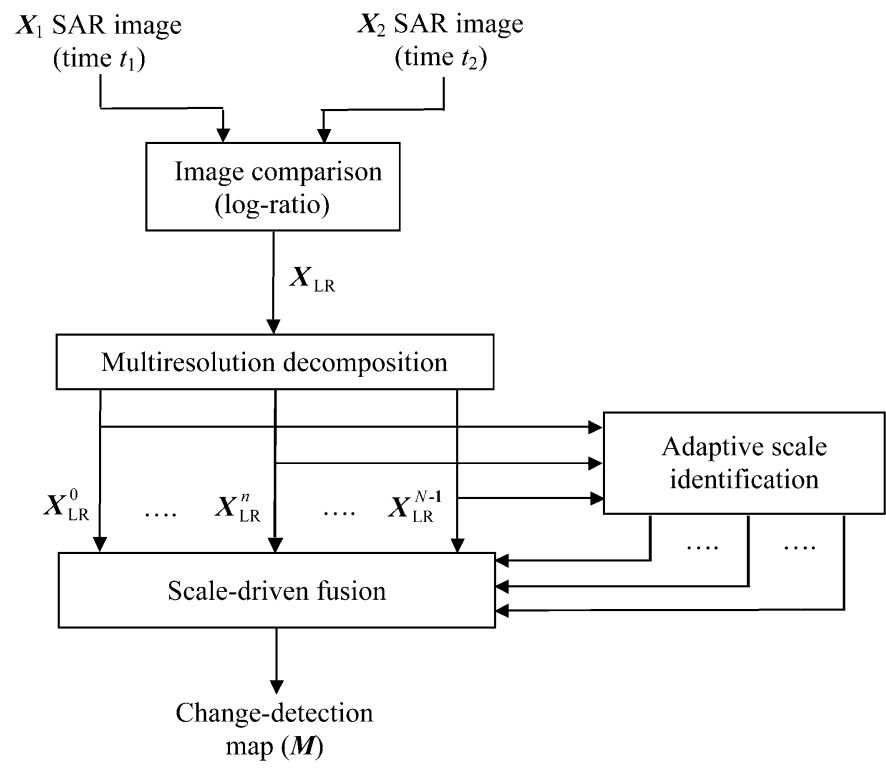

Fig. 1. General scheme of the proposed approach.

geometrical details (e.g., the borders of changed areas are well preserved) and a high robustness to noisy speckle components in homogeneous areas. Experimental results carried out on multitemporal European Remote Sensing 1 (ERS-1) satellite images from Canada confirm the effectiveness of the proposed scale-driven approach.

The paper is organized into five sections. The next section introduces the problem formulation and the basics of the proposed approach. Section III presents the proposed approach and focuses on its principal steps: i.e., 1) multiresolution decomposition; 2) adaptive scale identification; and 3) scale-driven fusion. Experimental results obtained on real multitemporal SAR data are reported in Section IV. Finally, conclusions are drawn in Section V.

\section{Problem Formulation And Architecture OF THE PROPOSED TECHNIQUE}

Let us consider the two coregistered intensity SAR images, $\boldsymbol{X}_{1}=\left\{X_{1}(i, j), 1 \leq i \leq I, 1 \leq j \leq J\right\}$ and $\boldsymbol{X}_{2}=$ $\left\{X_{2}(i, j), 1 \leq i \leq I, 1 \leq j \leq J\right\}$, of size $I \cdot J$, acquired over the same area at different times $t_{1}$ and $t_{2}$. Let $\Omega=\left\{\omega_{c}, \omega_{u}\right\}$ be the set of classes associated with changed and unchanged pixels. As shown in Fig. 1, the proposed approach is made up of four blocks aimed at the following: 1) image comparison; 2) multiresolution decomposition; 3 ) adaptive scale identification based on the local statistics computed at different resolution levels; and 4) generation of the final change-detection map according to an adaptive scale-driven fusion.

The first step of the proposed change-detection technique consists of a pixel-by-pixel image comparison. According to the literature [9], [17]-[19], the comparison is carried out by a ratio operator to reduce the effects of speckle in the resulting image and for the measured signal to be independent of the absolute intensity value of the considered pixel in the multitemporal images [18], [19]. Let $\boldsymbol{X}_{\mathrm{R}}$ (where the subscript $R$ stands for ratio) be the obtained "ratio image." To enhance low-intensity pixels, the ratio image is usually expressed in a logarithmic scale, resulting in the log-ratio image $\boldsymbol{X}_{\mathrm{LR}}$

$$
\boldsymbol{X}_{\mathrm{LR}}=\log \boldsymbol{X}_{\mathrm{R}}=\log \frac{\boldsymbol{X}_{2}}{\boldsymbol{X}_{1}}=\log \boldsymbol{X}_{2}-\log \boldsymbol{X}_{1}
$$

where $\log$ stands for natural-logarithm. With this operation the distribution of the two classes of interest $\left(\omega_{c}\right.$ and $\left.\omega_{u}\right)$ in the ratio image can be made more symmetrical and the residual multiplicative speckle noise can be transformed in an additive noise component [9]. ${ }^{1}$

The second step of the proposed method aims at building a multiscale representation of the change information in the considered test site. The desired scale-dependent representation can be obtained by applying different methods to the data, e.g., Laplacian/Gaussian pyramid decomposition [20], wavelet transform [21], [22], recursively upsampled bicubic filter [23], etc. Given the computational cost and the assumption of the additive noise model required by the above techniques, we chose to apply the multiresolution decomposition process to the log-ratio image $\boldsymbol{X}_{\mathrm{LR}}$, instead of decomposing the two original images $\boldsymbol{X}_{1}$ and $\boldsymbol{X}_{2}$ separately. At the same time this allows a reduction in computational cost and satisfies the additive noise model hypothesis. The selection of the most appropriate multiresolution technique is related to the statistical behaviors of $\mathbf{X}_{\mathrm{LR}}$ and will be discussed in the next section. The multiresolution decomposition step produces a set of images

$$
\mathbf{X}_{\mathrm{MS}}=\left\{\boldsymbol{X}_{\mathrm{LR}}^{0}, \ldots, \boldsymbol{X}_{\mathrm{LR}}^{n}, \ldots, \boldsymbol{X}_{\mathrm{LR}}^{N-1}\right\},
$$

where the superscript $n(n=0,1, \ldots, N-1)$ indicates the resolution level. As we shall consider a dyadic decomposition process, the scale corresponding to each resolution level is given by $2^{n}$. In our notation, the output at resolution level 0 corresponds to the original image, i.e., $\boldsymbol{X}_{\mathrm{LR}}^{0} \equiv \boldsymbol{X}_{\mathrm{LR}}$. For $n$ ranging from 0 to $N-1$, the obtained images are distinguished by different tradeoffs between spatial-detail preservation and speckle reduction. In particular, images with a low value of $n$ are strongly affected by speckle, but they are characterized by a large amount of geometrical detail, whereas images identified by a high value of $n$ show significant speckle reduction and contain degraded geometrical details (high frequencies are smoothed out).

In the third step, local and global statistics are evaluated for each pixel at different resolution levels. At each resolution level and for each spatial position, by comparing the local and global statistical behaviors it is possible to identify adaptively whether the considered scale is reliable for the analyzed pixel.

The selected scales are used to drive the fourth step, which consists of the generation of the change-detection map according to a scale-driven fusion. In this paper, three different scale-driven combination strategies are proposed and investigated. Two perform fusion at the decision level, while the third performs it at the feature level. Fusion at the decision level can either be based on "optimal" scale selection or on the use of all reliable scales; fusion at the feature level is carried out by analyzing all reliable scales.

\footnotetext{
${ }^{1}$ It is worth noting that the residual multiplicative speckle noise is expected to be particularly high in portions of the ratio image associated with changed areas on the ground.
} 


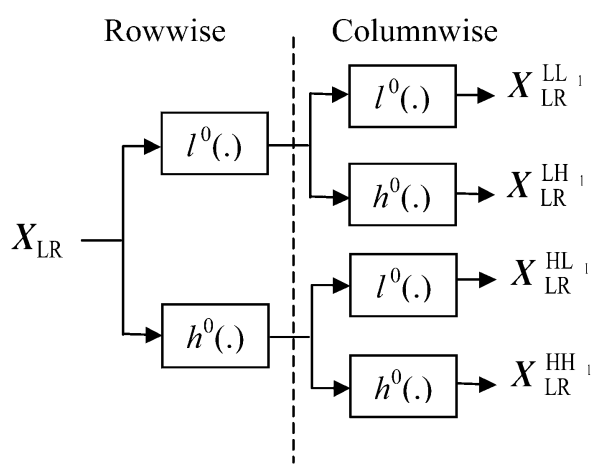

Fig. 2. Block scheme of the stationary wavelet decomposition of the log-ratio image $\boldsymbol{X}_{\mathrm{LR}}$.

\section{Proposed Adaptive SCALE-Driven Change-Detection Technique}

In this section, we focus on the novel aspects of the proposed change-detection approach. In particular, we present in detail the techniques for the following: 1) obtaining a multiresolution decomposition of the log-ratio image; 2) adaptively identifying the scales to be considered in the analysis of each pixel; and 3) producing the final change-detection map according to a scale-driven fusion strategy.

\section{A. Multiresolution Decomposition of the Log-Ratio Image}

As mentioned in the previous section, our aim is to handle the information at different scales (resolution levels) in order to improve both geometrical detail preservation and accuracy in homogeneous areas in the final change-detection map. Images included in the set $\mathbf{X}_{\mathrm{MS}}$ are computed by adopting a multiresolution decomposition process of the log-ratio image $\boldsymbol{X}_{\mathrm{LR}}$. In the SAR literature [15], [24]-[28], image multiresolution representation has been applied extensively to image denoising. Here, a decomposition based on the two-dimensional discrete stationary wavelet transform (2D-SWT) has been adopted, as in our image analysis framework it has a few advantages (as described in the following) over the standard discrete wavelet transform (DWT) [29]. As the log-ratio operation transforms the SAR signal multiplicative model into an additive noise model, SWT can be applied to $\boldsymbol{X}_{\mathrm{LR}}$ without any additional processing. By applying SWT to the log-ratio image instead of working separately on the two images $\boldsymbol{X}_{1}$ and $\boldsymbol{X}_{2}$ the computational time of the proposed change-detection technique can be halved. 2D-SWT applies appropriate level-dependent highand low-pass filters with impulse response $h^{n}($.$) and l^{n}($.$) ,$ $(n=0,1, \ldots, N-1)$, respectively, to the considered signal at each resolution level. A one-step wavelet decomposition is based on both level-dependent high- and low-pass filtering, first along rows and then along columns in order to produce four different images at the next scale. After each convolution step, unlike DWT, SWT avoids downsampling the filtered signals. Thus, according to the scheme in Fig. 2, the image $\boldsymbol{X}_{\mathrm{LR}}$ is decomposed into four images of the same size as the original. In particular, decomposition produces: 1) a lower resolution version $\boldsymbol{X}_{\mathrm{LR}}^{\mathrm{LL}_{1}}$ of image $\boldsymbol{X}_{\mathrm{LR}}$, which is called the approximation subband, and contains low spatial frequencies both in the horizontal and the vertical direction at resolution level 1; and 2) three high-frequency images $\boldsymbol{X}_{\mathrm{LR}}^{\mathrm{LH}_{1}}, \boldsymbol{X}_{\mathrm{LR}}^{\mathrm{HL}_{1}}$ and $\boldsymbol{X}_{\mathrm{LR}}^{\mathrm{HH}_{1}}$, which correspond to the horizontal, vertical, and diagonal detail subbands at resolution level 1, respectively. Note that, superscripts LL, LH, HL, and HH specify the order in which high- $(H)$ and low-pass $(L)$ filters have been applied to obtain the considered subband.

Multiresolution decomposition is obtained by recursively applying the described procedure to the approximation subband $\boldsymbol{X}_{\mathrm{LR} n}^{\mathrm{LL} n}$ obtained at each scale $2^{n}$. Thus, the outputs at a generic resolution level $n$ can be expressed analytically as follows:

$$
\begin{aligned}
& \boldsymbol{X}_{\mathrm{LR}}^{\mathrm{LL}(n+1)}(i, j)=\sum_{p=0}^{D^{n}-1} \sum_{q=0}^{D^{n}-1} l^{n}[p] l^{n}[q] \boldsymbol{X}_{\mathrm{LR}}^{\mathrm{LL} n}(i+p, j+q) \\
& \boldsymbol{X}_{\mathrm{LR}}^{\mathrm{LH}(n+1)}(i, j)=\sum_{p=0}^{D^{n}-1} \sum_{q=0}^{D^{n}-1} l^{n}[p] h^{n}[q] \boldsymbol{X}_{\mathrm{LR}}^{\mathrm{LL} n}(i+p, j+q) \\
& \boldsymbol{X}_{\mathrm{LR}}^{\mathrm{HL}(n+1)}(i, j)=\sum_{p=0}^{D^{n}-1} \sum_{q=0}^{D^{n}-1} h^{n}[p] l^{n}[q] \boldsymbol{X}_{\mathrm{LR}}^{\mathrm{LL} n}(i+p, j+q) \\
& \boldsymbol{X}_{\mathrm{LR}}^{\mathrm{HH}(n+1)}(i, j)=\sum_{p=0}^{D^{n}-1} \sum_{q=0}^{D^{n}-1} h^{n}[p] h^{n}[q] \boldsymbol{X}_{\mathrm{LR}}^{\mathrm{LL} n}(i+p, j+q)
\end{aligned}
$$

where $D^{n}$ is the length of the wavelet filters at resolution level $n$. At each decomposition step, the length of the impulse response of both high- and low-pass filters is upsampled by a factor 2 . Thus, filter coefficients for computing subbands at resolution level $n+1$ can be obtained by applying a dilation operation to the filter coefficients used to compute level $n$. In particular, $2^{n-1}$ zeros are inserted between the filter coefficients used to compute subbands at the lower resolution level [29]. This allows a reduction in the bandwidth of the filters by a factor two between subsequent resolution levels.

Filter coefficients of the first decomposition step for $n=0$ depend on the selected wavelet family and on the length of the chosen wavelet filter. According to an analysis of the literature [26], [30], we selected the Daubechies wavelet family and set the filter length to 8 . Dubechies of order 4 low-pass filter prototype impulse response is given by the following coefficient set:

$$
\{0.230378,0.714847,0.630881,-0.0279838,-0.187035 \text {, }
$$$$
0.0308414,0.0328830,-0.0105974\} \text {. }
$$

The finite impulse response of the high-pass filter for the decomposition step is obtained by satisfying the properties of the quadrature mirror filters. This is done by reversing the order of the low-pass decomposition filter coefficient and by changing the sign of the even indexed coefficients [31].

In order to adopt the proposed multiresolution fusion strategies, one should return to the original image domain. This is done by applying the two-dimensional inverse stationary wavelet transform (2D-ISWT) at each computed resolution level independently. For further detail about the stationary wavelet transform, the reader is referred to [29].

In order to obtain the desired image set $\mathbf{X}_{\mathrm{MS}}$ (where each image contains information at a different resolution level), for each resolution level a one step inverse stationary wavelet transform is applied in the reconstruction phase as many times as in 
the decomposition phase. The reconstruction process can be performed by applying the 2D-ISWT: 1) either to the approximation and thresholded detail subbands at the considered level (this is usually done in wavelet-based speckle filters [27]); or 2) only to the approximation subbands at each resolution level. ${ }^{2}$ Since the change-detection phase considers all the different levels, all the geometrical detail is in $\mathbf{X}_{\mathrm{MS}}$ even when detail coefficients at a particular scale are neglected (in other words, the details removed at a certain resolution level are recovered at a higher level without removing them from the decision process). For this reason, in this paper for simplicity we focus on the solution that only considers the approximation subbands in the reconstruction phase (it is worth noting that empirical experiments on real data have confirmed that details subband elimination does not affect the change-detection accuracy provided by the proposed approach). Once all resolution levels have been brought back to the image domain, the desired multiscale sequence of images $\boldsymbol{X}_{\mathrm{LR}}^{n}(n=0,1, \ldots, N-1)$ is complete and each element in $\mathbf{X}_{\mathrm{MS}}$ has the same size as the original image.

It is important to point out that unlike DWT, SWT avoids decimating. Thus, this multiresolution decomposition strategy "fills in the gaps" caused by the decimation step in the standard wavelet transform [29]. In particular, the SWT decomposition preserves translation invariance and allows avoiding aliasing effects during synthesis without providing high-frequency components.

\section{B. Adaptive Scale Identification}

Based on the obtained set of multiscale images $\boldsymbol{X}_{\mathrm{LR}}^{n}(n=$ $0,1, \ldots, N-1)$, we must identify reliable scales for each considered spatial position in order to drive the next fusion stage with this information. By using this information we can obtain change-detection maps characterized by high accuracy in homogeneous and border areas.

Reliable scales are selected according to whether the considered pixel belongs to a border or a homogeneous area at different scales. It is worth noting that the information at low-resolution levels is not reliable for pixels belonging to the border area, because at those scales details and edge information has been removed from the decomposition process. Thus, a generic scale is reliable for a given pixel, if the pixel at this scale is not in a border region or if it does not represent a geometrical detail.

To define whether a pixel belongs to a border or a homogeneous area at a given scale $n$, we propose to use a multiscale local coefficient of variation $\left(\mathrm{LCV}^{n}\right)$, as typically done in adaptive speckle denoising algorithms [28], [32]. This allows to better handle any residual multiplicative noise that may still be present in the scale selection process after rationing. ${ }^{3}$ As the coefficient of variation cannot be computed on the multiresolution log-ratio image sequence, the analysis is applied to the mul-

\footnotetext{
${ }^{2}$ It is worth noting that the approximation subband contains low frequencies in both horizontal and vertical directions. It represents the input image at a coarser scale and contains most informative components, whereas detail subbands contain information related to high frequencies (i.e., both geometrical detail information and noise components) each in a preferred direction. According to this observation, it is easy to understand how proper thresholding of detail coefficients allows noise reduction [27].

${ }^{3}$ An alternative choice could be to use the standard deviation computed on the log-ratio image. However, in this way we would neglect possible residual effects of the multiplicative noise component.
}

tiresolution ratio image sequence, which can easily be obtained from the former by inverting the logarithm operation. Furthermore, it should be mentioned that by working on the multiresolution ratio sequence we can design a homogeneity test capable of identifying border regions (or details) and no-border regions related to the presence of changes on the ground. This is different from applying the same test to the original images (which would result in identifying border and no-border regions with respect to the original scene but not with respect to the change signal).

The $\mathrm{LCV}^{n}$ is defined as

$$
\operatorname{LCV}^{n}(i, j)=\frac{\sigma^{n}(i, j)}{\mu^{n}(i, j)}
$$

where $\sigma^{n}(i, j)$ and $\mu^{n}(i, j)$ are the local standard deviation and the local mean, respectively, computed for the spatial position $(i, j)$ at resolution level $n(n=0,1, \ldots, N-1)$, on a moving window of a user-defined size. A window size that is too small reduces the reliability of the local statistical parameters, while windows that are too large decrease in sensitivity to identify geometrical details. Thus, the selected size should be a tradeoff between the above properties. Thanks to the normalization operation defined in (3), we can adapt the standard deviation to the multiplicative speckle model. This coefficient is a measure of the scene heterogeneity [32]: low values correspond to homogeneous areas, while high values refer to heterogeneous areas (e.g., border areas and point targets). To separate the homogeneous from the heterogeneous regions, a threshold value must be defined. In a homogeneous region the degree of homogeneity can be expressed in relation to the global coefficient of variation $\left(\mathrm{CV}^{n}\right)$ of the considered image at resolution level $n$, which is defined as

$$
\mathrm{CV}^{n}=\frac{\sigma^{n}}{\mu^{n}}
$$

where $\sigma^{n}$ and $\mu^{n}$ are the mean and the standard deviation computed over a homogeneous region at resolution level $n,(n=$ $0,1, \ldots, N-1)$. Homogeneous regions at each scale can be defined as those regions that satisfy the following condition:

$$
\mathrm{LCV}^{n}(i, j) \leq \mathrm{CV}^{n}
$$

In greater detail, a resolution level $r(r=0,1, \ldots, N-1)$ is said to be reliable for a given pixel if (5) is satisfied for all resolution levels $t(t=0,1, \ldots, r)$. Thus, for the pixel $(i, j)$, the set $\mathbf{X}_{\mathrm{MS}}^{R_{i j}}$ of images with reliable scale is defined as

$\mathbf{X}_{\mathrm{MS}}^{R_{i j}}=\left\{\boldsymbol{X}_{\mathrm{LR}}^{0}, \ldots, \boldsymbol{X}_{\mathrm{LR}}^{n}, \ldots, \boldsymbol{X}_{\mathrm{LR}}^{S_{i j}}\right\}, \quad$ with $S_{i j} \leq N-1$

where $S_{i j}$ is the level with the lowest resolution (identified by the highest value of $n$ ), such that the pixel can be represented without any border problems and therefore it satisfies the definition of reliable scale showed in (5) (note that the value of $S_{i j}$ is pixel dependent).

It is worth noting that, if the scene contains different kinds of changes with different radiometry (e.g., with increasing and decreasing radiometry), the above analysis should be applied to the normalized ratio image $\boldsymbol{X}_{\mathrm{NR}}$ (rather than to the standard ratio image $\boldsymbol{X}_{\mathrm{R}}$ ) defined as

$$
\boldsymbol{X}_{\mathrm{NR}}=\min \left\{\frac{\boldsymbol{X}_{1}}{\boldsymbol{X}_{2}}, \frac{\boldsymbol{X}_{2}}{\boldsymbol{X}_{1}}\right\}
$$


This makes the identification of border areas independent of the order with which the images are considered in the ratio, thus allowing all changed areas (independently of the related radiometry) to play a similar role in the definition of border pixels.

\section{Scale-Driven Fusion}

Once the set $\mathbf{X}_{\mathrm{MS}}^{R_{i j}}$ has been defined for each spatial position, it is possible to derive the final change-detection map according to a proper scale-driven fusion strategy. In this paper we propose and investigate three possible strategies: 1) fusion at the decision level by an "optimal" scale selection (FDL-OSS); 2) fusion at the decision level on all reliable scales (i.e., scales included in $\mathbf{X}_{\mathrm{MS}}^{R_{i j}}$ ) (FDL-ARS); and 3) fusion at the feature level on all reliable scales (FFL-ARS).

For each pixel, the fusion at the decision level by an "optimal" scale selection (FDL-OSS) strategy only considers the reliable level with the lowest resolution, i.e., the "optimal" resolution level $S_{i j}$. The rationale of this strategy is that the reliable level with the lowest resolution presents an "optimal" tradeoff between speckle reduction and detail preservation for the considered pixel. In greater detail, each scale-dependent image in the set $\mathbf{X}_{\mathrm{MS}}$ is analyzed independently in order to discriminate between the two classes $\omega_{c}$ and $\omega_{u}$ associated with change and no-change classes, respectively. The desired partitioning for the generic scale $n$ can be obtained by thresholding $\boldsymbol{X}_{\mathrm{LR}}^{n}$. It is worth noting that since the threshold value is scale dependent, given the set of images $\mathbf{X}_{\mathrm{MS}}=$ $\left\{\boldsymbol{X}_{\mathrm{LR}}^{0}, \ldots, \boldsymbol{X}_{\mathrm{LR}}^{n}, \ldots, \boldsymbol{X}_{\mathrm{LR}}^{N-1}\right\}$, we should determine (either automatically [9], [33], [34] or manually) a set of threshold values $T=\left\{T^{0}, \ldots, T^{n}, \ldots, T^{N-1}\right\}$. Regardless of the threshold-selection method adopted, a sequence of change-detection maps $\mathbf{M}_{\mathrm{MS}}=\left\{\boldsymbol{M}^{0}, \ldots, \boldsymbol{M}^{n}, \ldots, \boldsymbol{M}^{N-1}\right\}$ is obtained from the images in $\mathbf{X}_{\mathrm{MS}}=\left\{\boldsymbol{X}_{\mathrm{LR}}^{0}, \ldots, \boldsymbol{X}_{\mathrm{LR}}^{n}, \ldots, \boldsymbol{X}_{\mathrm{LR}}^{N-1}\right\}$. A generic pixel $M(i, j)$ in the final change-detection map $\boldsymbol{M}$ is assigned to the class it belongs to in the map $\boldsymbol{M}^{S_{i j}}\left(\in \mathbf{M}_{\mathrm{MS}}\right)$ computed at its optimal selected scale $S_{i j}$, i.e.,

$$
\begin{aligned}
& M(i, j) \in \omega_{k} \Leftrightarrow M^{S_{i j}}(i, j) \in \omega_{k}, \\
& \quad \text { with } k=\{c, u\} \text { and } S_{i j} \leq N-1 .
\end{aligned}
$$

The accuracy of the resulting change-detection map depends both on the accuracy of the maps in the multiresolution sequence and on the effectiveness of the procedure adopted to select the optimal resolution level. Both aspects are affected by the amount of residual noise in $\boldsymbol{X}_{\mathrm{LR}}^{S_{i j}}$.

To make the decision process more robust to noise, we propose an alternative approach that considers all reliable changedetection maps (with respect to the scale of the pixel) and applies a fusion at decision level rule (FDL-ARS). For each pixel, the set $M_{\mathrm{MS}}^{R}(i, j)=\left\{M^{0}(i, j), \ldots, M^{n}(i, j), \ldots, M^{S_{i j}}(i, j)\right\}$ of the related reliable multiresolution labels is considered. Each label $M^{n}$ in $M_{\mathrm{MS}}^{R}$ can be seen as a decision of a member of a pool of experts. Thus, the pixel is assigned to the class that obtains the highest number of votes. In actual fact, the final change-detection map $\boldsymbol{M}$ is computed by applying at each spatial position a majority voting rule to the set $M_{\mathrm{MS}}^{R}(i, j)$. The class that receives the largest number of votes $V_{\omega_{k}}(i, j), k=$

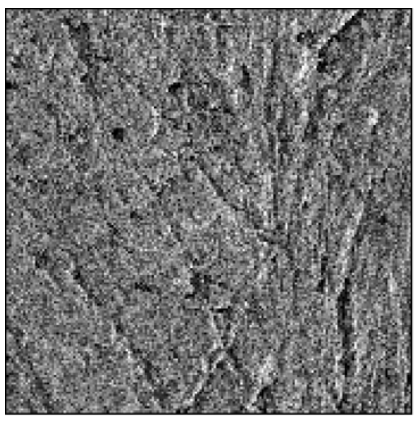

(a)

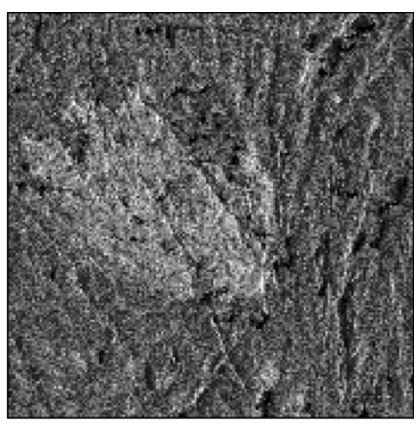

(b)

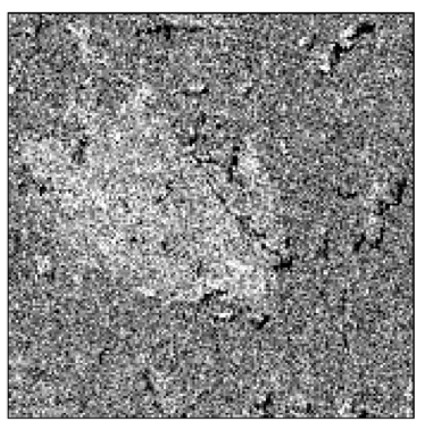

(c)

Fig. 3. Images of the Saskatchewan province, Canada, used in the experiments. (a) Image acquired from the ERS-1 SAR sensor in July 1995 (b) Image acquired from the ERS-1 SAR sensor in October 1995. (c) Analyzed log-ratio image.

$\{c, u\}$, represents the final decision for the considered input pattern, i.e.,

$$
M(i, j) \in \omega_{k} \Leftrightarrow \omega_{k}=\underset{\omega_{h} \in \Omega}{\operatorname{argmax}}\left\{V_{\omega_{h}}(i, j)\right\}, k=\{c, u\} .
$$

The main disadvantage of the FDL-ARS strategy is that it only considers the final classification of each pixel at different reliable scales. A better exploitation of the information in the multiresolution sequence $\mathbf{X}_{\mathrm{MS}}$ can be obtained by considering a fusion at feature-level strategy (FFL-ARS). In order to accomplish the fusion process at different scales, a new set of images $\overline{\mathbf{X}}_{\mathrm{MS}}^{R}=\left\{\overline{\boldsymbol{X}}_{\mathrm{MS}}^{0}, \ldots, \overline{\boldsymbol{X}}_{\mathrm{MS}}^{n}, \ldots, \overline{\boldsymbol{X}}_{\mathrm{MS}}^{N-1}\right\}$ is computed by averaging all possible sequential combinations of images in $\mathbf{X}_{\mathrm{MS}}$, i.e.,

$$
\overline{\boldsymbol{X}}_{\mathrm{MS}}^{n}=\frac{1}{n+1} \sum_{h=0}^{n} \boldsymbol{X}_{\mathrm{LR}}^{h}, \quad n=0,1, \ldots, N-1
$$

where the superscript $n$ identifies the highest scale included in the average operation. When low values of $n$ are considered, the image $\overline{\boldsymbol{X}}_{\mathrm{MS}}^{n}$ contains a large amount both of geometrical details and of speckle components, whereas when $n$ increases, the image $\overline{\boldsymbol{X}}_{\mathrm{MS}}^{n}$ contains a smaller amount both of geometrical details and of speckle components. A pixel in position $(i, j)$ is assigned to the class obtained by applying a standard thresholding procedure to the image $\overline{\boldsymbol{X}}_{\mathrm{MS}}^{S_{i j}}, S_{i j} \leq N-1$, computed by averaging on the reliable scales selected for that spatial position, i.e.,

$$
M(i, j) \in \begin{cases}\omega_{u}, & \text { if } \overline{\boldsymbol{X}}_{\mathrm{MS}}^{S_{i j}}(i, j) \leq T^{S_{i j}} \\ \omega_{c}, & \text { if } \overline{\boldsymbol{X}}_{\mathrm{MS}}^{S_{i j}}(i, j)>T^{S_{i j}}\end{cases}
$$




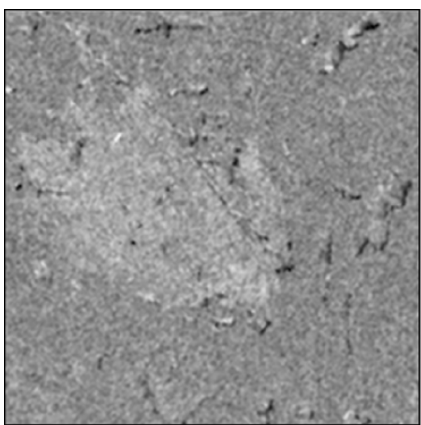

(a)

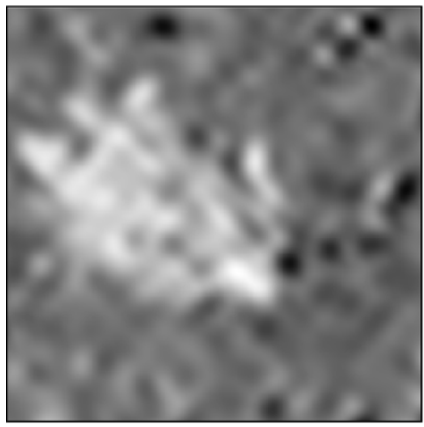

(d)

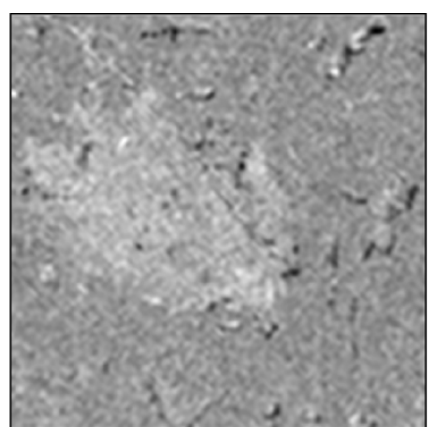

(b)

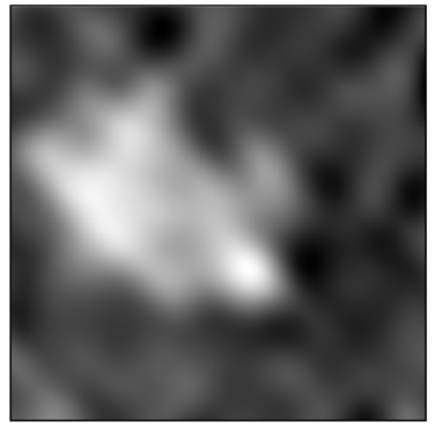

(e)

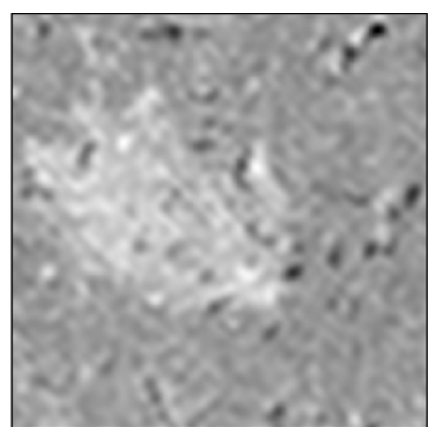

(c)

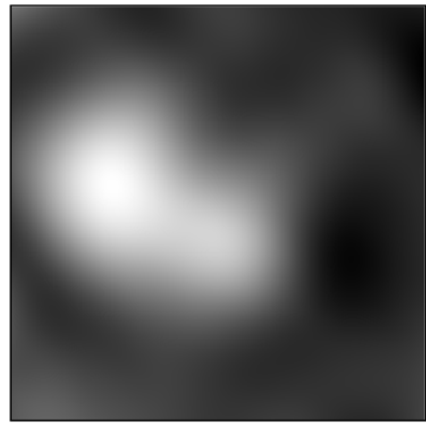

(f)

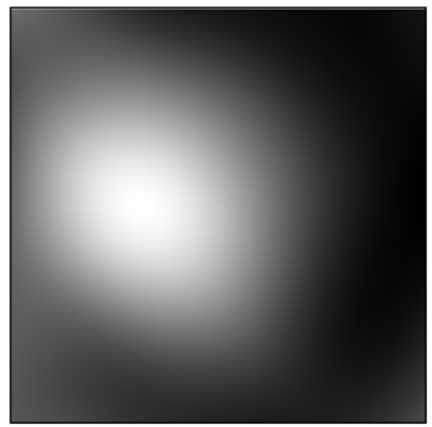

(g)

Fig. 4. Multiscale image sequence obtained by applying the wavelet decomposition procedure to the log-ratio image $\mathbf{X}_{\mathrm{MS}}=\left\{\boldsymbol{X}_{\mathrm{LR}}^{1}, \ldots, \boldsymbol{X}_{\mathrm{LR}}^{7}\right\}$.

where $T^{S_{i j}}$ is the decision threshold optimized (either automatically [9], [33], [34] or manually) for the considered image $\overline{\boldsymbol{X}}_{\mathrm{MS}}^{S_{i j}}$. The latter strategy is expected to be capable of exploiting also the information component in the speckle, as it considers all the high frequencies in the decision process. It is worth noting that in the FFL-ARS strategy, as the information present at a given scale $r$ is also contained in all images $\boldsymbol{X}_{\mathrm{LR}}^{n}$ with $n<r$, in the fusion process the components characterizing the optimal scale $S_{i j}$ (and the scales closer to the optimal one) are implicitly associated with greater weights than those associated with other considered levels. This seems reasonable, given the importance of these components for the analyzed spatial position.

\section{EXPERIMENTAL RESULTS}

\section{A. Dataset Description}

The dataset used in the experiments is made up of two SAR images acquired by the ERS-1 SAR sensor (C-band and VV-polarization) in the province of Saskatchewan (Canada) before (July 1) and after (October 14) the 1995 fire season. The two considered images are characterized by a geometrical resolution of $25[\mathrm{~m}]$ in both directions and by a nominal number of looks equal to 5. The selected test site [see Fig. 3(a) and (b)] is a section $(350 \times 350$ pixels $)$ of the entire available scene. A fire caused by a lightning event destroyed a large portion of the vegetation in the considered area between the two aforementioned dates.

The two multilook intensity images were geocoded using the digital elevation model (DEM) GTOPO30; no speckle reduction algorithms were applied to the images. The log-ratio image was computed from the above data according to (1).

In order to be able to make a quantitative evaluation of the effectiveness of the proposed approach, a reference map was defined manually [see Fig. 5(b)]. To this end, we used the available ground-truth information provided by the Canadian Forest Service (CFS) and by the fire agencies of the individual Canadian provinces. Ground truth information is coded in a vector format and includes information about fires (e.g., geographical coordinates, final size, cause, etc.) occurred from 1981 to 1995 and greater than 200 ha in final size. CFS ground truth was used for a rough localization of the burned areas as it shows a medium 
TABLE I

Overall Error, False Alarms, and Missed Alarms (in Number of Pixels and Percentage) RESUlting From the Proposed AdAPTIVE SCALE-DrivEN Fusion APPROACHES

\begin{tabular}{c|c|c|c|c|c|c|c}
\hline \multirow{2}{*}{ Fusion strategy } & \multicolumn{2}{|c|}{ False Alarms } & \multicolumn{2}{c|}{ Missed Alarms } & \multicolumn{2}{c|}{ Overall Errors } & \multirow{2}{*}{ LCV window size } \\
\cline { 2 - 7 } & Pixels & $\mathbf{\%}$ & Pixels & $\mathbf{\%}$ & Pixels & \% & \\
\hline FDL-OSS & 3791 & $3.75 \%$ & 3812 & $17.91 \%$ & 7603 & $6.21 \%$ & $23 \times 23$ \\
\hline FDL-ARS & 2695 & $2.66 \%$ & 3528 & $16.58 \%$ & 6223 & $5.08 \%$ & $7 \times 7$ \\
\hline FFL-ARS & 2181 & $2.15 \%$ & 3376 & $15.86 \%$ & 5557 & $4.54 \%$ & $5 \times 5$ \\
\hline
\end{tabular}

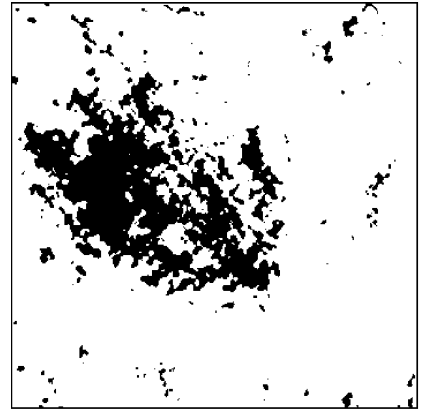

(a)

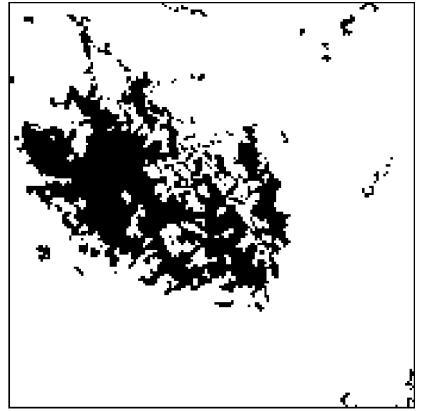

(b)
Fig. 5. (a) Change-detection map obtained for the considered dataset using the FFL-ARS strategy on all reliable scales. (b) Reference map of the changed area used in the experiment.

geometrical resolution. An accurate identification of the boundaries of the burned areas was obtained from a detailed visual analysis of the two original five-look intensity images [Fig. 3(a) and (b)], the ratio image and the log-ratio image [Fig. 3(c)] carried out accurately in cooperation with experts in SAR-image interpretation. In particular, different color composites of the above-mentioned images were used to highlight all the portions of the changed areas in the best possible way. It is worth noting that no despeckling or wavelet based analysis was applied to the images exploited to generate the reference map for this process to be as independent as possible of the methods adopted in the proposed change-detection technique. In generating the reference map, the irregularities of the edges of the burned areas were faithfully reproduced in order to be able to make an accurate assessment of the effectiveness of the proposed change-detection approach. At the end of the process, the obtained reference map contained 101219 unchanged pixels and 21281 changed pixels. Our goal was to obtain, with the proposed automatic technique, a change-detection map as similar as possible to the reference map obtained according to the aforementioned time-consuming manual process driven with ground truth information and by experts in SAR image interpretation.

\section{B. Results}

Several experiments were carried out to assess the effectiveness of the proposed change-detection technique (which is based on scale-driven fusion strategies) with respect to classical methods (which are based on thresholding of the log-ratio image).

In all trials involving image thresholding, the optimal threshold value was obtained according to a manual trial-and-error procedure. In greater detail, (among all possible values) we selected for each image the threshold value that showed the minimum overall error in the change-detection map compared to the reference map. Thanks to this it was possible to evaluate the optimal performance of the proposed methodology without any bias due to human operator subjectivity or to the fact that the selection was made by an automatic thresholding algorithm. However, any type of automatic threshold-selection technique can be used with this technique (see [9] for more details about automatic thresholding of the log-ratio image). As the described procedure is independently optimized for each considered image, it leads to different threshold values in each case. Performance assessment was accomplished both quantitatively (in terms of overall errors, false and missed alarms) and qualitatively (according to a visual comparison of the produced change-detection maps with reference data).

In order to apply the three proposed scale-driven fusion strategies (see Section III), the log-ratio image was first decomposed into seven resolution levels by applying the Daubechies-4 wavelet transform. Each computed approximation subband was used to construct different scales, i.e., $\mathbf{X}_{\mathrm{MS}}=\left\{\boldsymbol{X}_{\mathrm{LR}}^{1}, \ldots, \boldsymbol{X}_{\mathrm{LR}}^{7}\right\}$ (see Fig. 4). In order to avoid distortions introduced along image borders by the SWT, the multiresolution wavelet decomposition was applied to a log-ratio image larger than $350 \times 350$ pixels in the accuracy assessment phase. It is worth noting that the full-resolution original image $\boldsymbol{X}_{\mathrm{LR}}\left(\equiv \boldsymbol{X}_{\mathrm{LR}}^{0}\right)$ was discarded from the analyzed set, since it was affected by a strong speckle noise. In particular, empirical experiments pointed out that when $\boldsymbol{X}_{\mathrm{LR}}^{0}$ is used on this dataset the accuracy of the proposed change-detection technique gets degraded. Nevertheless, in the general case, resolution level 0 can also be considered and should not be discarded a priori. A number of trials were carried out to identify the optimal window size to compute the local coefficient of variation $\left(\mathrm{LCV}^{n}\right)$ used to detect detail pixels (e.g., border) at different resolution levels. The optimal size (i.e., the one that gives the minimum overall error) was selected for all analyzed strategies (see Table I).

Table I summarizes the quantitative results obtained with the different fusion strategies proposed. As can be seen from the analysis of the overall error, the FFL-ARS strategy gave the lowest error, i.e., 5557 pixels, while the FDL-ARS strategy gave 6223, and the FDL-OSS strategy 7603 (the highest overall error). As expected, by including all the reliable scales in the fusion phase it was possible to improve the change-detection accuracy compared to a single "optimal" scale. In greater detail, the FFL-ARS strategy gave the lowest false and missed alarms, decreasing their values by 1610 and 436 pixels, respectively, compared to the FDL-OSS strategy. This is because on the one hand the FDL-OSS procedure is penalized both by the change-detection accuracy at a single resolution level (which is 
TABLE II

Overall Error, False Alarms, and Missed Alarms (in Number of PiXels and Percentage) RESUlting From Classical CHANGE-DETECTION APPROACHES

\begin{tabular}{l|c|c|c|c|c|c|c}
\hline \multirow{2}{*}{$\begin{array}{c}\text { Applied filtering } \\
\text { technique }\end{array}$} & \multicolumn{2}{|c|}{ False Alarms } & \multicolumn{2}{c|}{ Missed Alarms } & \multicolumn{2}{c|}{ Total Errors } & \multirow{2}{*}{ Filter window size } \\
\cline { 2 - 8 } & Pixels & $\%$ & Pixels & $\%$ & Pixels & $\%$ & \\
\hline Enhanced Lee filter & 3725 & $3.68 \%$ & 4328 & $20.34 \%$ & 8053 & $6.57 \%$ & $7 \times 7$ \\
\hline Gamma MAP filter & 3511 & $3.47 \%$ & 4539 & $21.33 \%$ & 8050 & $6.57 \%$ & $7 \times 7$ \\
\hline Wavelet denoising & 2769 & $2.74 \%$ & 4243 & $19.94 \%$ & 7012 & $5.72 \%$ & - \\
\hline
\end{tabular}

significantly affected by noise when fine scales are considered) and by residual errors in identifying the optimal scale of a given pixel; on the other hand, because the use of the entire subset of reliable scales allows a better exploitation of the information at the highest resolution levels of the multiresolution sequence in the change-detection process. It is worth noting that FFL-ARS outperformed FDL-ARS also in terms of false (2181 versus 2695) and missed (3376 versus 3528) alarms. This is mainly due to its ability to better handle all the information in the scale-dependent images before the decision process. This leads to a more accurate recognition of critical pixels (i.e., pixels that are very close to the boundary between the changed and unchanged classes on the log-ratio image), that exploit the joint consideration of all the information present at the different scales in the decision process. For a better understanding of the results achieved, we made a visual analysis of the obtained change-detection maps. Fig. 5(a) shows the change-detection map obtained with the FFL-ARS strategy (which proved to be the most accurate), while Fig. 5(b) is the reference map. As can be seen, the considered strategy produced a change-detection map that was very similar to the reference map. In particular, the change-detection map obtained with the proposed approach shows good properties both in terms of detail preservation and in terms of high accuracy in homogeneous areas.

In order to assess the effectiveness of the proposed scale-driven change-detection approach, the results obtained with the FFL-ARS strategy were compared with those obtained with a classical change-detection algorithm. In particular, we computed a change-detection map by an optimal (in the sense of minimum error) thresholding of the log-ratio image obtained after despeckling with the adaptive enhanced Lee filter [32]. The enhanced Lee filter was applied to the two original images (since a multiplicative speckle model is required). Several trials were carried out while varying the window size, in order to find the value that leads to the minimum overall error. The best result for the considered test site (see Table II) was obtained with a $7 \times 7$ window size. The thresholding operation gave an overall error of 8053 pixels. This value is significantly higher than the overall error obtained with the FFL-ARS strategy (i.e., 5557). In addition the proposed scale-driven fusion technique also decreased both the false (2181 versus 3725 ) and the missed alarms (3376 versus 4328) compared to the considered classical procedure. From a visual analysis of Fig. 6(a) and Fig. 5(a) and (b), it is clear that the change-detection map obtained after the Lee-based despeckling procedure significantly reduces the geometrical detail content in the final change-detection map compared to that obtained with the FFL-ARS approach. This is mainly due to the use of the filter, which not only results

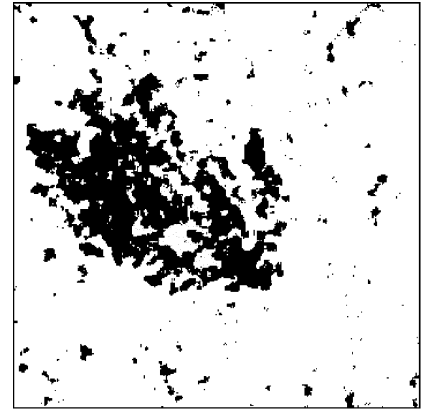

(a)

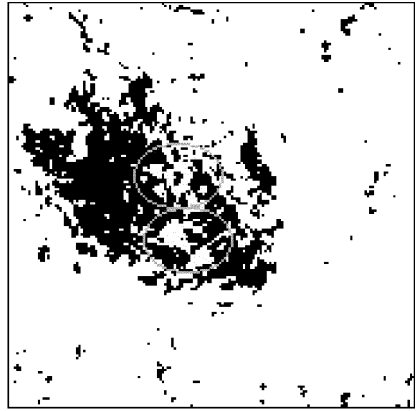

(b)
Fig. 6. Change-detection maps obtained for the considered dataset by optimal manual thresholding of the log-ratio image after the despeckling with (a) the Lee-enhanced filter and (b) the DWT-based technique.

in a significant smoothing of the images but also strongly reduces the information component present in the speckle. Similar results and considerations both from a quantitative and qualitative point of view were obtained by filtering the image with the Gamma MAP filter (compare Tables I and II).

To better understand the extent of the validity of the proposed scale-driven method, we also analyzed the effectiveness of classical thresholding of the log-ratio image after denoising with a recently proposed more advanced despeckling procedure. In particular, we investigated a discrete wavelet transform based denoising [27], [35] technique (not used previously in change-detection problems). This technique achieves noise reduction in three steps: 1) image decomposition (DWT); 2) thresholding of wavelet coefficients; and 3) image reconstruction by inverse wavelet transformation (IDWT) [27], [35]. It is worth noting that also this procedure is based on the multiscale decomposition of the images. We can, therefore, better evaluate the effectiveness of the scale-driven procedure in exploiting the multiscale information obtained with the DWT decomposition. Wavelet based denoising was applied to the log-ratio image since an additive speckle model is required. Several trials were carried out varying the wavelet-coefficient denoising algorithm while keeping the type of wavelet fixed, i.e., Daubechies-4 (the same used for multilevel decomposition). The best result (see Table II) was obtained by a soft thresholding of the detail coefficients according to the universal threshold $T=\sqrt{2 \sigma^{2} \log (I \cdot J)}$, where $I \cdot J$ is the image size and $\sigma^{2}$ is the estimated noise variance [35]. The soft thresholding procedure sets detail coefficients that fall between $T$ and $-T$ to zero, and shrinks the module of coefficients that fall out of this interval by a factor $T$. The noise variance estimation was performed by computing the variance of the diagonal-detail subband at the first decomposition level. Given the above thresholding 
approach, we selected the number of decomposition levels that resulted in the minimum change-detection overall error. This minimum was reached with a six-level wavelet decomposition. However, also in this case the obtained error (i.e., 7012 pixels) was significantly higher than the overall error obtained with the proposed approach based on the FFL-ARS strategy (i.e., 5557 pixels). Moreover, the presented method performed better also in terms of false and missed alarms, which were reduced from 4243 to 3376, and from 2769 to 2181, respectively (see Tables I and II). By analyzing Fig. 6(b), 5(a), and 5(b), it can be seen that the change-detection map obtained by thresholding the log-ratio image after applying the DWT-based denoising algorithm preserves geometrical information well. Nevertheless, on observing the map in greater detail, it can be concluded qualitatively that the spatial fidelity obtained with this procedure is lower than that obtained with the proposed approach. This is confirmed, for example, when we look at the right part of the burned area [circles in Fig. 6(b)], where some highly irregular areas saved from the fire are properly modeled by the proposed technique, but smoothed out by the procedure based on DWT denoising. This confirms the quantitative results and thus the effectiveness of the proposed approach in exploiting information from multilevel image decomposition.

It is worth noting that the improvement in performance shown by the proposed approach was obtained without any additional computational burden compared to the thresholding procedure after wavelet denoising. In particular, both methods require analysis and synthesis steps (though for different purposes). The main difference between the two considered techniques is the scale-driven combination step, which does not increase the computational time required by the thresholding of detail coefficients according to the standard wavelet-based denoising procedure.

\section{Discussion AND CONClusion}

In this paper, a novel adaptive scale-driven approach to change detection in multitemporal SAR images has been proposed. Unlike classical methods, this approach exploits information at different scales (obtained by a wavelet-based decomposition of the log-ratio image) in order to improve the accuracy and geometric fidelity of the change-detection map.

Three different fusion strategies that exploit the subset of reliable scales for each pixel have been proposed and tested: 1) fusion at the decision level by an optimal scale selection (FDL-OSS); 2) fusion at the decision level of all reliable scales (FDL-ARS); and 3) fusion at the feature level of all reliable scales (FFL-ARS). As expected, a comparison among these strategies showed that fusion at the feature level led to better results than the other two procedures, in terms both of geometrical detail preservation and accuracy in homogeneous areas. This is due to a better intrinsic capability of this technique to exploit the information present in all the reliable scales for the analyzed spatial position, including the amount of information present in the speckle.

Experimental results confirmed the effectiveness of the proposed scale-driven approach with the FFL-ARS strategy on the considered dataset. This approach outperformed a classical change-detection technique based on the thresholding of the log-ratio image after a proper despeckling based on the application of the enhanced Lee filter and also of the Gamma filter. In particular, change detection after despeckling resulted in a higher overall error, more false alarms and missed alarms, and significantly lower geometrical fidelity. In order to further assess the validity of the proposed approach, the standard technique based on the thresholding of the log-ratio image was applied after a despeckling phase applied according to an advanced DWT-based denoising procedure (which has not been used previously in change-detection problems). The obtained results suggest that the proposed approach performs slightly better in terms of spatial fidelity and significantly increases the overall accuracy of the change-detection map. This confirms that on the considered dataset and for solving change-detection problems, the scale-driven fusion strategy exploits the multiscale decomposition better than standard denoising methods.

As a final remark, it is worth noting that all experimental results were carried out applying an optimal manual trial-and-error threshold selection procedure, in order to avoid any bias related to the selected automatic procedure in assessing the effectiveness of both the proposed and standard techniques. Nevertheless, this step can be performed adopting automatic thresholding procedures [9], [36].

Future developments of this work are related to the application of the proposed adaptive scale-driven approach to change detection in very high-resolution SAR images. Furthermore, we plan to extend the use of the scale-driven technique to change detection in multiband and fully polarimetric SAR data.

\section{ACKNOWLEDGMENT}

The authors are grateful to F. Holecz and P. Pasquali (SARMAP s.a., Switzerland) for providing images of Canada and advice about ground truth, and to the anonymous referees for their constructive criticism.

\section{REFERENCES}

[1] P. S. Chavez, Jr and D. J. Mackinnon, "Automatic detection of vegetation changes in the southwestern United States using remotely sensed images," Photogramm. Eng. Remote Sens., vol. 60, no. 5, pp. 1285-1294, May 1994.

[2] J. Cihlar, T. J. Pultz, and A. L. Gray, "Change detection with synthetic aperture radar," Int. J. Remote Sens., vol. 13, no. 3, pp. 401-414, 1992.

[3] T. Hame, I. Heiler, and J. San Miguel-Ayanz, "An unsupervised change detection and recognition system for forestry," Int. J. Remote Sens., vol. 19, no. 6, pp. 1079-1099, Apr. 1998.

[4] S. Quegan, T. Le Toan, J. J. Yu, F. Ribbes, and N. Floury, "Multitemporal ERS SAR analysis applied to forest mapping," IEEE Trans. Geosci. Remote Sens., vol. 38, no. 2, pp. 741-753, Mar. 2000.

[5] K. Grover, S. Quegan, and C. da Costa Freitas, "Quantitative estimation of tropical forest cover by SAR," IEEE Trans. Geosci. Remote Sens., vol. 37, no. 1, pp. 479-490, Jan. 1999.

[6] L. Bruzzone and S. B. Serpico, "An iterative technique for the detection of land-cover transitions in multispectral remote-sensing images," IEEE Trans. Geosci. Remote Sens., vol. 35, no. 4, pp. 858-867, Jul. 1997.

[7] K. R. Merril and L. Jiajun, "A comparison of four algorithms for change detection in an urban environment," Remote Sens. Environ., vol. 63, no. 2, pp. 95-100, Feb. 1998.

[8] T. Thuy Lee and R. Florrence, "Rice crop and monitoring using ERS-1 data based on experiment and modeling results," IEEE Trans. Geosci. Remote Sens., vol. 35, no. 1, pp. 41-55, Jan. 1997.

[9] Y. Bazi, L. Bruzzone, and F. Melgani, "An unsupervised approach based on the generalized Gaussian model to automatic change detection in multitemporal SAR images," IEEE Trans. Geosci. Remote Sens., vol. 43, no. 4, pp. 874-887, Apr. 2005. 
[10] V. S. Frost, J. A. Stiles, K. S. Shanmungan, and J. C. Holtzman, “A model for radar images and its application to adaptive digital filtering of multiplicative noise," IEEE Trans. Pattern Anal. Mach. Intell., vol. PAMI-4, no. 2, pp. 157-165, Mar. 1982.

[11] J. S. Lee, "Digital image enhancement and noise filtering by use of local statistics," IEEE Trans. Pattern Anal. Mach. Intell., vol. PAMI-2, no. 2, pp. 165-168, Mar. 1980.

[12] D. T. Kuan, A. A. Sawchuk, T. C. Strand, and P. Chavel, "Adaptive noise smoothing filter for images with signal-dependent noise," IEEE Trans. Pattern Anal. Mach. Intell., vol. PAMI-7, no. 2, pp. 165-177, Mar. 1985.

[13] A. Lopes, E. Nerzy, R. Touzi, and H. Laur, "Structure detection and statistical adaptive filtering in SAR images," Int. J. Remote Sens., vol. 41, pp. 1735-1758, 1993.

[14] — "Maximum a posteriori speckle filtering and first order texture models in SAR images," in Proc. IGARSS, vol. 3, College Park, MD, May 20-24, 1990, pp. 2409-2412.

[15] S. Solbø and T. Eltoft, " $\Gamma$-WMAP: A statistical speckle filter operating in the wavelet domain," Int. J. Remote Sens., vol. 25, no. 5, pp. 1019-1036, Mar. 2004.

[16] W. Dierking and H. Skriver, "Change detection for thematic mapping by means of airborne multitemporal polarimetric SAR imagery," IEEE Trans. Geosci. Remote Sens., vol. 40, no. 3, pp. 618-636, Mar. 2002.

[17] R. J. Dekker, "Speckle filtering in satellite SAR change detection imagery," Int. J. Remote Sens., vol. 19, no. 6, pp. 1133-1146, 1998.

[18] E. J. M. Rignot and J. J. van Zyl, "Change detection techniques for ERS-1 SAR data,” IEEE Trans. Geosci. Remote Sens., vol. 31, no. 4, pp. 896-906, Jul. 1993.

[19] C. J. Oliver and S. Quegan, Understanding Synthetic Aperture Radar Images. Norwood, MA: Artech House, 1998.

[20] P. J. Burt and E. H. Adelson, "The Laplacian pyramid as a compact image code," IEEE Trans. Commun., vol. COM-31, no. 4, pp. 532-540, Apr. 1983.

[21] S. G. Mallat, "A theory for multiresolution signal decomposition: The wavelet representation," IEEE Trans. Pattern Anal. Mach. Intell., vol. PAMI-11, no. 7, pp. 674-693, Jul. 1989.

[22] - A Wavelet Tour of Signal Processing. San Diego, CA: Academic, 1998.

[23] B. Aiazzi, L. Alparone, S. Baronti, V. Cappellini, R. Carlà, and L. Mortelli, "A Laplacian pyramid with rational scale factor for multisensor image data fusion," in Proc. Int. Conf. Sampling Theory and Applications-SampTA 97, 1997, pp. 55-60.

[24] B. Aiazzi, L. Alparone, and S. Baronti, "Multiresolution local-statistics speckle filtering based on a ratio Laplacian pyramid," IEEE Trans. Geosci. Remote Sens., vol. 36, no. 5, pp. 1466-1476, Sep. 1998.

[25] H. Xie, L. E. Pierce, and F. T. Ulaby, "SAR speckle reduction using wavelet denoising and Markov random field modeling," IEEE Trans. Geosci. Remote Sens., vol. 40, no. 10, pp. 2196-2212, Oct. 2002.

[26] Z. Zeng and I. Cumming, "Bayesian speckle noise reduction using the discrete wavelet transform," in Proc. IGARSS, Seattle, WA, Jul. 06-10, 1998, pp. 7-9.

[27] H. Guo, J. E. Odegard, M. Lang, R. A. Gopinath, I. W. Selesnick, and C. S. Burrus, "Wavelet based speckle reduction with application to SAR based ATD/R," in Proc. IEEE Int. Conf. Image Processing, vol. 1, 1994, pp. 75-79.

[28] F. Argenti and L. Alparone, "Speckle removal for SAR images in the undecimated wavelet domain," IEEE Trans. Geosci. Remote Sens., vol. 40, no. 11, pp. 2363-2374, Nov. 2002.

[29] G. P. Nason and B. W. Silverman, "The stationary wavelet transform and some statistical applications," in Wavelets and Statistics, A. Antoniadis and G. Oppenheim, Eds. New York: Springer-Verlag, 1995, vol. 103, Lecture Notes Statistics, pp. 281-300.

[30] V. Manian and R. Vàsquez, "On the use of transform for SAR image classification," in Proc. IGARSS, vol. 2, Jul. 6-10, 1998, pp. 1068-1070.

[31] G. Strang and T. Nguyen, Wavelets and Filter Banks. Reading, MA: Wellesley-Cambridge, 1996.

[32] A. Lopes, R. Touzi, and E. Nerzy, "Adaptive speckle filters and scene heterogeneity," IEEE Trans. Geosci. Remote Sens., vol. 28, no. 6, pp. 992-1000, Nov. 1990.

[33] R. O. Duda, P. E. Hart, and D. G. Stork, Pattern Classification. New York: Wiley, 2001.

[34] F. Melgani, G. Moser, and S. B. Serpico, "Unsupervised change-detection methods for remote-sensing data," Opt. Eng., vol. 41, no. 12, pp. 3288-3297, Dec. 2002.
[35] D. L. Donoho and I. M. Johnstone, "Ideal spatial adaptation via wavelet shrinkage," Biometrika, vol. 81, pp. 425-455, 1994.

[36] L. Bruzzone and D. Fernández Prieto, "An adaptive semiparametric and context-based approach to unsupervised change detection in multitemporal remote-sensing images," IEEE Trans. Image Process., vol. 11, no. 4, pp. 446-452, Apr. 2002.

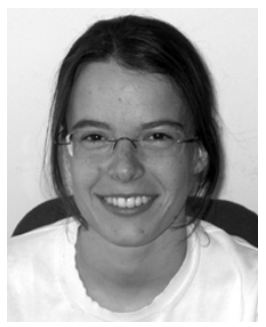

Francesca Bovolo received the laurea (B.S.) and laurea specialistica (M.S.) degrees in telecommunication engineering (summa cum laude) from the University of Trento, Trento, Italy, in 2001 and 2003, respectively. She is currently pursuing the Ph.D. degree in information and communication technologies at the University of Trento.

She is presently with the Pattern Recognition and Remote Sensing Group, Department of Information and Communication Technologies, University of Trento. Her main research activity is in the area of remote sensing image processing. In particular, her interests are related to change detection in multispectral and SAR images. She conducts research on these topics within the frameworks of several national and international projects.

Ms. Bovolo is a referee for the IEEE TRANSACTIONS ON GEOSCIENCE AND REMOTE SENSING.

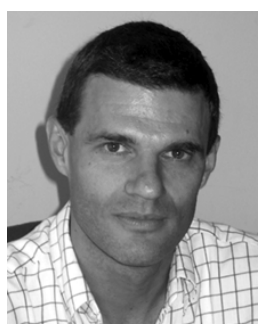

Lorenzo Bruzzone (S'95-M'99-SM'03) received the laurea (M.S.) degree in electronic engineering (summa cum laude) and the Ph.D. degree in telecommunications from the University of Genoa, Genoa, Italy, in 1993 and 1998, respectively.

He is currently Head of the Remote Sensing Laboratory in the Department of Information and Communication Technologies at the University of Trento, Trento, Italy. From 1998 to 2000, he was a Postdoctoral Researcher at the University of Genoa. From 2000 to 2001, he was an Assistant Professor at the University of Trento, and from 2001 to February 2005 he was an Associate Professor of telecommunications at the same university. Since March 2005, he has been a Full Professor of telecommunications at the University of Trento, where he currently teaches remote sensing, pattern recognition, and electrical communications. His current research interests are in the area of remote sensing image processing and recognition (analysis of multitemporal data, feature selection, classification, regression, data fusion, and neural networks). He conducts and supervises research on these topics within the frameworks of several national and international projects. He is the author (or coauthor) of more than 140 scientific publications, including journals, book chapters, and conference proceedings. He is a referee for many international journals and has served on the Scientific Committees of several international conferences. He is a member of the Scientific Committee of the India-Italy Center for Advanced Research.

Dr. Bruzzone ranked first place in the Student Prize Paper Competition of the 1998 IEEE International Geoscience and Remote Sensing Symposium (Seattle, July 1998). He was a recipient of the Recognition of IEEE Transactions on Geoscience and Remote Sensing Best Reviewers in 1999 and was a Guest Editor of a Special Issue of the IEEE TRANSACTIONS ON GEOSCIENCE AND REMOTE SENSING on the subject of the analysis of multitemporal remote sensing images (November 2003). He was the General Chair and Co-chair of the First and Second, respectively, IEEE International Workshop on the Analysis of Multitemporal Remote-Sensing Images. Since 2003, he has been the Chair of the SPIE Conference on Image and Signal Processing for Remote Sensing. From 2004 to 2005, he was an Associate Editor of the IEEE GEOSCIENCE AND REMOTE SENSING LETTERS. Since 2005, he has been an Associate Editor of the IEEE TRANSACTIONS ON GEOSCIENCE AND REMOTE SENSING. He is a member of the International Association for Pattern Recognition (IAPR) and of the Italian Association for Remote Sensing (AIT). 